\title{
Development and application of the WRFDA-Chem 3DVAR system: aiming to improve air quality forecast and diagnose model deficiencies
}

\author{
Wei Sun ${ }^{1.2}$, Zhiquan Liu" ${ }^{\prime}$, Dan Chen"s, Pusheng Zhao', and Min Chen \\ ' National Center for Atmospheric Research, Boulder, CO, 80301, USA \\ ${ }^{2}$ National Space Science Center, Chinese Academy of Sciences, Beijing, 100190, China \\ Institute of Urban Meteorology, China Meteorology Administration, Beijing, 100089, China
}

1. Bias for $\mathrm{PM}_{10}$ in NODA run

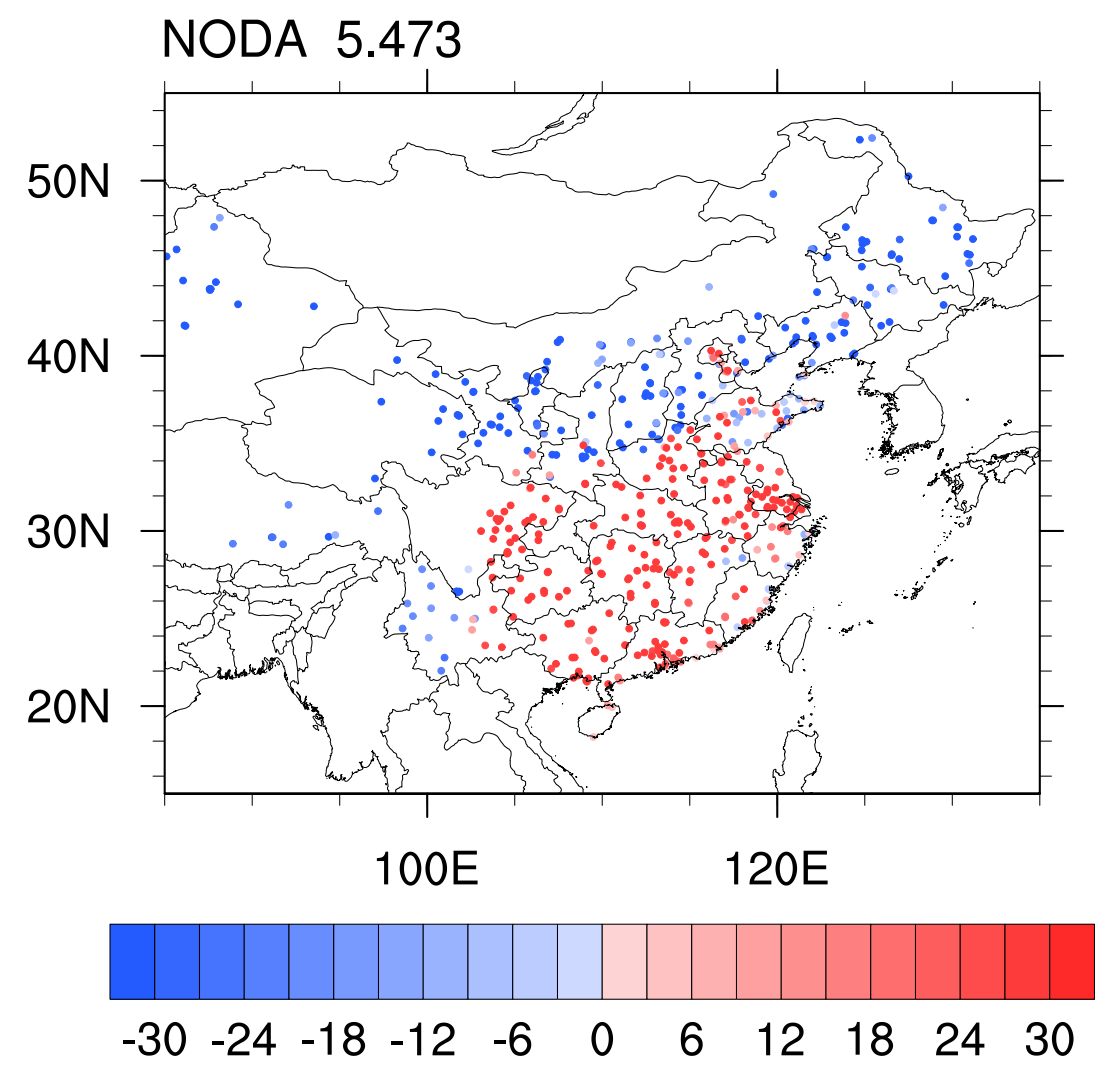

Fig. 1 Bias for PM10 at 531 stations in NODA experiment.

*Corresponding author:Dr.Zhiquan Liu (liuz@ucar.edu) and Dr.Dan Chen (dchen@ium.cn) 
2. Threat score for air quality index

The threat score (TS) for air quality index (AQI) is calculated by

$$
T S_{i}=\frac{H_{i}}{H_{i}+M_{i}+F_{i}}
$$

where $\mathrm{H}, \mathrm{M}$, and $\mathrm{F}$ denotes the times of the hits, the misses, and the false alarms in the forecast of AQI, and $\mathrm{i}$ denotes the AQI levels from 1 to 6. In result, the TS is acquired at each AQI level ranging from 0 to 1 . The higher (lower) TS represents the better (worse) forecast performance. 